\title{
OBSERVATIONS OF INTERSTELLAR CARBON COMPOUNDS
}

\author{
E. Dartois ${ }^{1}$
}

\begin{abstract}
Infrared absorption and emission features observed spectroscopically in our Galaxy allow to probe the composition of solid dust grains, their evolution and thus follow the cycling of matter in the Galaxy. Many observables do reveal the presence of large amounts of carbonaceous particles in space, other than the PAH-like emission lines. The carbonaceous materials observed include amorphous carbons, diamondoids showing in emission for a few specific sources, and the recently detected fullerenes. An important hydrogenated amorphous carbon component (HAC or a-C:H), traced by the $2940 \mathrm{~cm}^{-1}$ structured absorption feature is observed against Galactic background sources. Since the discovery of this feature in the early eighties (Allen 1981), the observation of a-C:H has been extended to the mid-infrared by space observatories, giving insight into additional associated features. They are also observed in external galaxies, showing the ubiquitous nature of these components. We will focus on astronomical observations of organic matter other than PAHs, amorphous carbons and associated laboratory dust analogues relevant to astrophysical applications.
\end{abstract}

\section{Introduction}

The nowdays observed dust results from the superposition and mixing of many differents galactic environments that participate to the so-called "dust lifecycle". Dust is produced in several source environments and, as it travels through the galaxy, experiences various physical and chemical processes that will modify its structure and/or composition. Among carbonaceous contributors, additional carbon allotropes, other than the polycyclic aromatic hydrocarbons (PAHs) described abundantly in this volume, participate in the building of the dusty interstellar medium. Organic matter includes various observed structural forms such as amorphous carbons, diamond, hydrogenated amorphous carbons and other materials with aromatic/aliphatic mixed structures.

\footnotetext{
1 Institut d'Astrophysique Spatiale, UMR-8617, Université Paris-Sud, Bâtiment 121, 91405 Orsay, France
} 


\section{Amorphous carbons}

Dust composition in the vicinity of evolved stars flows is constrained from direct observations and modelling. When the stellar wind flowing out reaches a pressure still sufficiently high $\left(10^{-5}-10^{-3} \mathrm{~Pa}\right)$ and temperature sufficiently low $(\sim 2500-$ $1000 \mathrm{~K}$ ), atoms and molecules nucleate small dust seeds that start to grow in the expanding flow, until they reach a region where the pressure and temperature regime does not allow to pursue an efficient grain growth (e.g. Patzer et al. 2003; Gail \& Sedlmayr 1999). This dust formation window is the consequence of the competition between the dust formation timescale and the stellar wind ejection speed that freezes the reactivity at some point. Passed this point in the flow, the dust formed leaves the nucleation regime and enters the evolution one. The kind of dust formed is controlled by chemical parameters such as the carbon/oxygen ratio in the flow. The observed circumstellar dust near evolved stars is a mix of oxygen and carbon rich dust. Of particular interest for the carbonaceous world are the socalled "carbon" stars, injecting important amounts of carbon under an amorphous form difficult to observe remotely, as no specific features are associated with them, unlike silicates. This allotrope is clearly observed around extreme carbon stars, as a broad featureless emission in equilibrium with the radiation field (See Fig. 1).
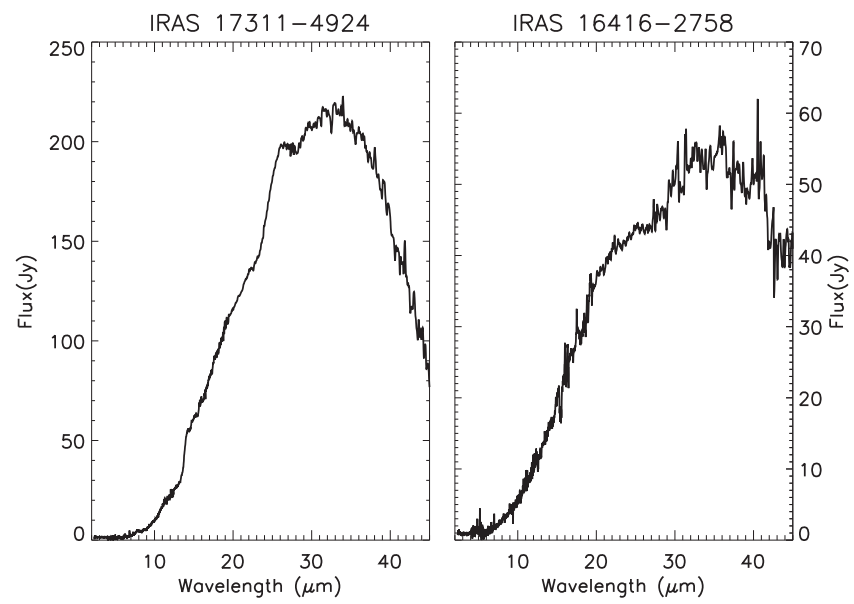

Fig. 1. Emission spectra of two carbon stars extracted from the Infrared Space Observatory database (http://iso.esac.esa.int/ida/). They illustrate the almost featureless continuum emission by highly carbonaceous grains in evolved carbonaceous stellar flows (see also Volk et al. 2001; Hony 2002; Chen et al. 2010 and citations therein).

These stars are likely to be the progenitors of C-rich protoplanetary nebulae (PPNs) such as AFGL 2688 (classified as a Class C by Peeters et al. 2002; see also Peeters in this volume), and C-rich planetary nebulae (PNs) such as NGC 7027. They will be involved in the carbon dust cycle although the grains they produced are more difficult to observe in the later phases. 


\section{3 (Nano-)diamonds}

The presence of high amounts of (nano-)diamonds, up to several thousands ppm (e.g. Ott 1993; Anders \& Zinner 1993), in the organic extracts from meteorites has led to the search for their plausible infrared signatures in the laboratory, to confront their spectra to those of young stellar objects observed in early evolution phases eventually leading to a protosolar system. They were initially proposed as an alternative explanation for some of the unidentified infrared bands (UIBs) and probably responsible for the "21 microns" astronomical feature, following infrared spectroscopy studies of "Allende" and "Orgueil" meteorite nanodiamonds (Koike et al. 1995; Hill et al. 1998).

The unambiguous detection of specific $\mathrm{CH}$ methine and methylene stretching modes, observed at 3.53 and $3.43 \mu \mathrm{m}$, associated with hydrogen atoms chemically bounded to diamond structure was demonstrated by Guillois et al. (1999), by comparing emission spectra of astronomical sources to laboratory spectra of nanocrystal diamond films exposed to hydrogen by Chang et al. (1995). These sources are different from the ones previously discussed for the 21 "microns" feature. Recently, from a molecular approach, Pirali et al. (2007) measured small diamondoids in the gas phase and, reported calculations, as well as attenuated total reflectance spectra for higher diamondoids. Analogues of the measured species with the same symmetry containing around $130 \mathrm{C}$ atoms $\left(\right.$ e.g., $\mathrm{C}_{136} \mathrm{H}_{104}$ ) show an intensity ratio of the 3.53 and $3.43 \mu \mathrm{m}$ bands close to that observed in the interstellar spectra. The inferred size is close to typical sizes of meteoritic diamonds.

Observationally, a survey of $30 \mathrm{Herbig}$ Ae/Be stars, the spectral type of the previously observed stars where the features had been observed, was conducted by Acke \& van den Ancker (2006). No new source could be added to the list of diamond emission features, implying that less than $4 \%$ of the Herbig targets show the prominent emission features at 3.43 and/or $3.53 \mu \mathrm{m}$.

In an effort to resolve spatially the location of these features, Habart et al. (2004) marginally resolved the emission intensity profile, showing that it is less extended than the associated $3.3 \mu \mathrm{m}$ PAH feature. Goto et al. (2009), using an adaptive optics system, resolved spatially the $\mathrm{PAH}$ and diamond $\mathrm{CH}$ emissions. The diamond emission is concentrated, peaking at about $30 \mathrm{AU}$ from the star. The PAH emission is more extended (above $100 \mathrm{AU}$ ). The authors speculate on diamond formation in these circumstellar disks, with graphitic grains processed into diamond grains under highly-energetic particle bombardment.

\section{Fullerenes}

Fullerenes and in particular $\mathrm{C}_{60}$ have been searched for intensively via electronic and infrared specific transitions (e.g. Foing \& Ehrenfreund 1994; Fulara et al. 1993; Moutou et al. 1999; Herbig 2000). $\mathrm{C}_{60}$ was recently detected by Sellgren et al. $(2009,2010)$ and Cami et al. (2010). More details can be found in the contribution by K. Sellgren et al. in this volume. 


\section{Hydrogenated amorphous carbons}

Hydrogenated amorphous carbons, $\mathrm{HAC}$ or a-C:H for amorphous material made of $\mathrm{C}$ and $\mathrm{H}$, constitute one important component of interstellar dust. They were observed initially at $3.4 \mu \mathrm{m}$ against a Galactic center source (Allen \& Wickramasenghe 1981). The features contributing to this absorption band were early associated to $\mathrm{sp}^{3} \mathrm{CH}_{3}$ and $\mathrm{CH}_{2}$ stretching modes, as stated in e.g. Duley \& Williams (1983), "Interstellar amorphous carbon dust with chemisorbed $\mathrm{CH}_{2}$ and $\mathrm{CH}_{3}$ groups may be a significant component of interstellar dust in diffuse clouds". Since then, numerous experiments/observations have been performed to constrain its origin (e.g. Jones et al. 1983; Butchart et al. 1986; Mc Fadzean et al. 1989; Ehrenfreund et al. 1991; Sandford et al. 1991; Sandford et al. 1995; Pendleton et al. 1994; Tielens et al. 1996; Geballe et al. 1998; Chiar et al. 2002; Mennella et al. 2002; Pendleton \& Allamandola 2002). A Galactic abundance for a-C:H has been estimated from observed $\mathrm{CH}$ stretching modes. Depending on the assumed materials, the associated oscillator strength for $\mathrm{CH}$ modes and the degree of hydrogenation, the implied cosmic carbon fraction varies from $2.6 \%$ to $35 \%$ (Sandford et al. 1991), above 2.5 to $4 \%$ based on the spectra of alkanes (Pendleton et al. 1994) and up to 20-30\% for laboratory analogues of a-C:H (Duley 1994; Duley et al. 1998).

The constrain on a-C:H was initially set only by the $\mathrm{CH}$ stretching modes. Nevertheless, the band profiles of many laboratory analogues were already incompatible with the astronomical absorption profile. It is incompatible with $\mathrm{H}_{2} \mathrm{O}$ dominated photolytic or radiolysis residues obtained starting with an interstellar ice mantle composition (Bernstein et al. 1995; Allamandola et al. 1988; Greenberg et al. 1995; Pendleton \& Allamandola 2002). Instead, laboratory analogues using heated carbon rod (Schnaiter et al. 1998), laser desorbed carbon (Mennella et al. 1999), plasma deposition (Lee \& Wdowiak 1993; Furton et al. 1999) or photoproduced a-C:H at low temperature (Dartois et al. 2005) provide a much better fit to the observed features. The Insoluble Organic Matter (IOM) extracted from meteorites also provides a reasonable fit to the astronomical observations (e.g. Ehrenfreund et al. 1991) at $3.4 \mu \mathrm{m}$.

The discrimination became clearer with the advent of space telescopes, allowing to track the mid-infrared fingerprints of this material. The moderate to high oxygen content in many of the laboratory analogues or IOM does provide strong absorptions in the mid-IR fingerprint region, and the carbonyl band is one of the strongest in the 1650 to $1800 \mathrm{~cm}^{-1}$ range. Within the signal-to-noise achieved, these absorptions can at most be a minor contribution to the observed diffuse medium spectra, as shown in Figure 2. For Galactic sources there still exists confusion in the mid-infrared, due to foreground dense clouds ice absorptions (Fig. 2 upper spectra, Dartois et al. 2004), or local circumstellar contributions decoupled from the diffuse medium features (Fig. 2 lower spectra, Chiar \& Tielens 2001; Chiar et al. 2002). However, meaningful upper limits were already set.

In the last years, several extragalactic obscured active galactic nuclei (AGN) sources displaying a-C:H absorptions have been observed, mainly via the stretching 


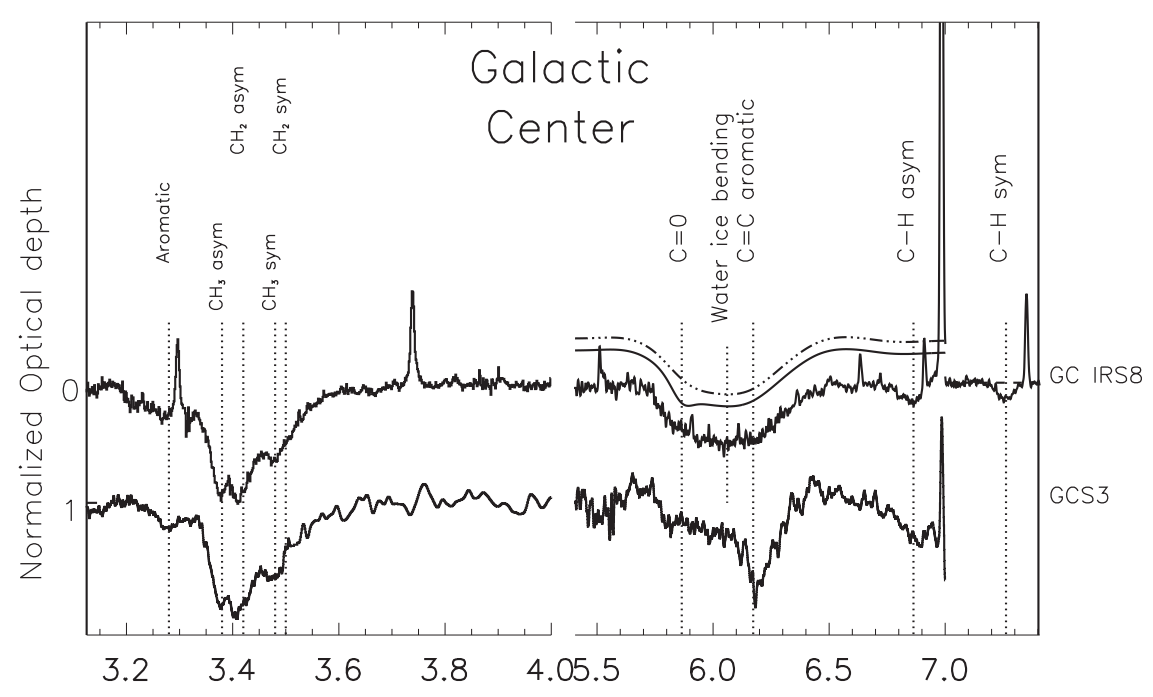

Fig. 2. Continuum extracted transmittance spectra along two Galactic center lines of sight. Both observations supposedly probe the same diffuse interstellar medium absorptions, but display many additional contributions arising from circumstellar and/or dense cloud contributions. Nevertheless, they constrain the mid-infrared counterpart of the $3.4 \mu \mathrm{m}$ absorption from a-C:H. See text for the detailed analysis of these differences.

modes (Pendleton et al. 1994; Mason et al. 2004; Dartois et al. 2004; Imanishi 2006; Risaliti et al. 2006; Imanishi et al. 2008). In NGC 1068, the hydrocarbon distribution has even been imaged to better constrain its spatial distribution, and foresee its nature (Mason et al. 2006; Geballe et al. 2009). The extragalactic sources provide a different constrain to get insight into the a-C:H structure and ubiquity. Contrary to Galactic embedded sources used as infrared background to probe the diffuse medium, obscured AGNs probe large dust column densities in front of an extended infrared continuum provided by the active nucleus environement. With a parsec-scale infrared probing pencil, the risk of very local circumstellar contamination is minimised. With a favorable AGN nucleus to diffuse medium alignment geometry, a line-of-sight free from significant dense cloud ice absorptions can be observed, providing a clean mid-infrared continuum to probe a-C:H fingerprints. Another advantage of the extragalactic case is the moderate galaxy red-shift, shifting the wavelength frame to more favourable atmospheric windows for ground-based observations, thus giving a unique access to the aromatic $\mathrm{CH}$ stretching mode region, devoid of strong telluric contamination, which are especially important for shallow absorption features.

The IRAS $08572+3915$ AGN is such a test case source, with a redshift of $\mathrm{z} \sim 0.0583$. An upper limit on the aromatic $\mathrm{C}-\mathrm{H}$ stretch has been evaluated, and the aromatic versus aliphatic $\mathrm{C}-\mathrm{H}$ content of interstellar a-C:H can be better constrained $(\mathrm{N}(\mathrm{CH}$ aromatic $) / \mathrm{N}(\mathrm{CH} \mathrm{sp} 3) \leq 0.08$; Dartois et al. 2007). Using the 
$\mathrm{N}(\mathrm{H})$ column density relation derived from the observed silicate optical depth combined to an elemental cosmic carbon abundance, about $15 \%$ of the cosmic $\mathrm{C}$ must be involved in $\mathrm{sp}^{3}$ bonding. The methyl and methylene $\mathrm{C}-\mathrm{H}$ observed ratio imply also $\mathrm{x}(\mathrm{H}) / \mathrm{x}\left(\mathrm{C} \mathrm{sp}^{3}\right) \sim 2.33$. Taking into account that only the infrared moderately active modes are observed, this sets a lower limit to the hydrogen content of the material observed, meaning a fractional hydrogen content in the a-C:H of $\mathrm{x}(\mathrm{H}) \geq 0.2$. These constraints can be brought together in a ternary phase diagram where the hydrogen content and the two main bonding types for carbon $\left(\mathrm{sp}^{2}\right.$ and $\mathrm{sp}^{3}$, as the sp contribution is expected to be small) constitute the poles (Ferrari \& Robertson 2000; Dartois et al. 2007). Overplotted are the constraints for IRAS $08572+3915$, as well as the position for different laboratory produced analogues, including soot, and PAHs lying on the $\mathrm{H}$ to $\mathrm{sp}^{2}$ border. Random Covalent Network models for materials made with olefinic or aromatic $\mathrm{C}=\mathrm{C}$, using the model developped by Angus \& Jansen (1988) and extended to aromatic structure by Jones (1990) are displayed. The a-C:H analogues fit perfectly with these constraints as well as the overall infrared spectrum.

In the model structure proposed by Pendleton \& Allamandola (2002, Figure 17 in this paper), the aromatic $\mathrm{CH}$ component ( $\sim 160$ by number) represents more than two times the aliphatic $\mathrm{CH}$ component $\left(\mathrm{CH}_{2}+\mathrm{CH}_{3} \sim 70\right)$, for a carbonaceous backbone of $\sim 540 \mathrm{C}$ atoms. We would expect in principle, with such a structure, an aromatic $\mathrm{CH}$ absorption higher than observed for IRAS $08572+3915$, even if the lower oscillator strength of the $\mathrm{CH}$ aromatic stretch is taken into account.

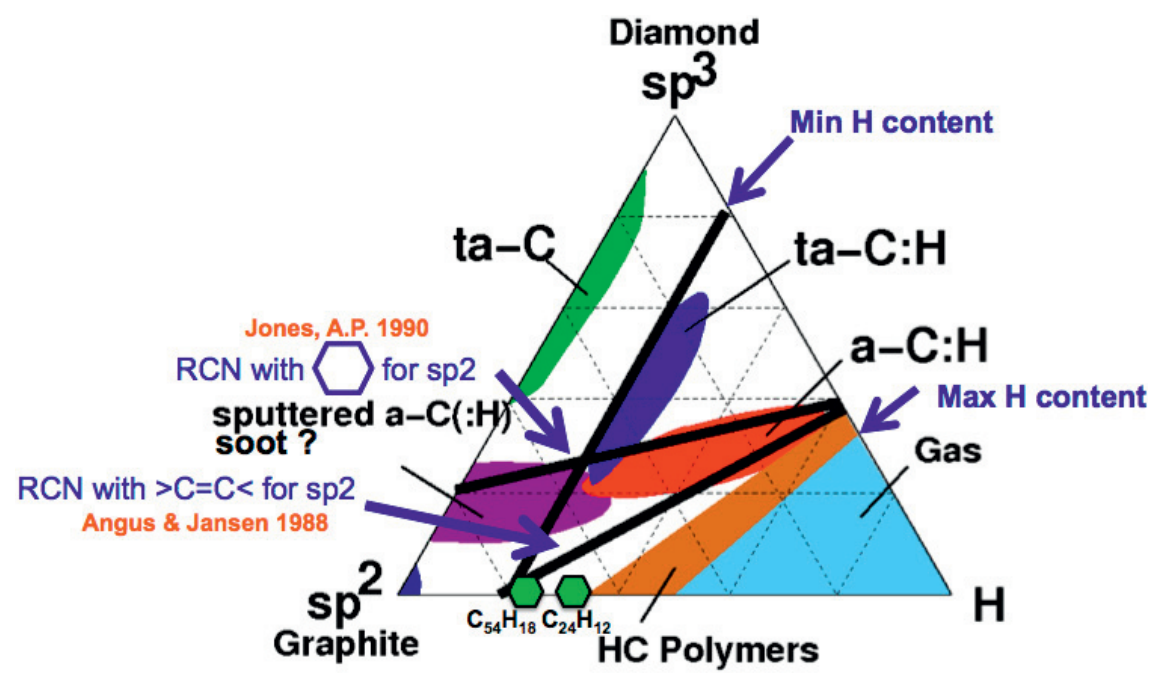

Fig. 3. Ternary phase diagram for analogues made principally from $\mathrm{C}$ and $\mathrm{H}$. The constrain from observations of IRAS $08572+3915$ is set (min H and max H contents). Several domains for various synthetic materials from the laboratory are shown. Two Random Covalent Network models (Angus \& Jansen 1988; Jones 1990) are also displayed, almost enclosing the a-C:H domain. 
Based on the observations and ternary diagram, we propose to revise the basic structural unit (BSU) issued from the Pendleton \& Allamandola analysis by using a material with a lower aromatic $\mathrm{CH}$ contribution ${ }^{1}$, such as hydrogenated amorphous carbon or hydrogen rich soot like analogues.

Interstellar a-C:H materials remain difficult to observe as compared to PAHs. Their astrophysical detection requires large column densities, as shown by the visual to band extinction relations $\left(\mathrm{A}_{\mathrm{V}} / \tau(3.4 \mu \mathrm{m}) \sim 250 ;\right.$ e.g. Sandford et al. 1995; $\mathrm{A}_{\mathrm{V}} / \tau(6.85 \mu \mathrm{m}) \sim 640$; Dartois \& Munoz Caro 2007). They represent a major ISM component, containing up to $30 \%$ of total galactic ISM carbon (typical $\sim 15 \%$ ). Their spectroscopic absorption feature contrast is easily hidden by PAHs when energetic photons are present, as strongly emitting $\mathrm{PAH}$ regions require only few tenth of an $A_{V}$ to be able to record bright emission bands. This explains why their observation is hampered in e.g. the interstellar media of Starburst galaxies. It is ubiquitous in the Galactic diffuse ISM and observed in a large number of external galaxies (e.g. Dartois \& Munoz-Caro 2007). It contains low amount of $\mathrm{O}$ heteroatoms. It is in agreement with laboratory plasma produced (microwave, laser) and/or photoproduced a-C:Hs. It may be partly responsible for ISM fluorescence features (e.g. Godard \& Dartois 2010 and references therein). It is a possible precursor for another form of interstellar carbon (PAHs) upon energetic processing.

\section{Mixed aliphatic/aromatic structural units}

\subsection{Observed classes $A, B, C$}

The relative profile variations of the mid-IR emission among various astrophysical sources has been underlined since a long time from ground-based observations (Joblin et al. 1996; Geballe et al. 1997) and a classification in various classes was performed when space missions provided a full wavelength infrared coverage (Peeters et al. 2002; van Diedenhoven et al. 2004, see Peeters elsewhere in this volume). Some of the observed sources, the so-called "class C", display both aromatic and aliphatic emission bands, questioning us on the possible link between hydrocarbons seen in absorption and emission. In few instances, the C-H dust stretching mode is spatially resolved in emission by adaptive optics (Goto et al. 2003; Goto et al. 2007) and relative variations from the central star suggest a progressive disappearance of aliphatic character to the benefit of the aromatic feature when the distance from the star increases. It has been shown that laboratory analogues thermally annealed at different temperatures, provide a reasonable match to this process (e.g. 2005, 2000, 1997, 1990). However one should take into account the possibility to have different emission mechanisms between both phases (solid and molecular), that will depend also on dust particles size in the stellar field. One must not forget that concomittant mechanisms are at work (chemical

\footnotetext{
${ }^{1}$ Note that the constrain is on the aromatic $\mathrm{CH}$. An aromatic backbone structure can still be present but interconnected to other elements via e.g. methylene or carbon-carbon bonds like in a-C:H or soot.
} 
modification, emission, stellar versus ISRF photon irradiations) that still need to be further constrained.

A progressive evolution among sources from class A to $\mathrm{C}$ has been notably underlined by Szczerba et al. (2005). The aromatic C $=\mathrm{C}$ stretching mode position is shown to shift from 6.2 to $6.3 \mu \mathrm{m}$ while the so-called "7.7" feature evolves toward $8 \mu \mathrm{m}$. The change in the $6.2 \mu \mathrm{m}$ feature position has been associated with various hypothesis, such as the formation of PAH metal complexes, the formation of PAH clusters, isotopic shifts, size changes or induced by heteroatoms like nitrogen. In the last case, the band would shift with $\mathrm{N}$ atom substitution site within the aromatic structure (Hudgins et al. 2005). In the following a simple alternative scenario is proposed to this evolution, based on recent laboratory experiments.
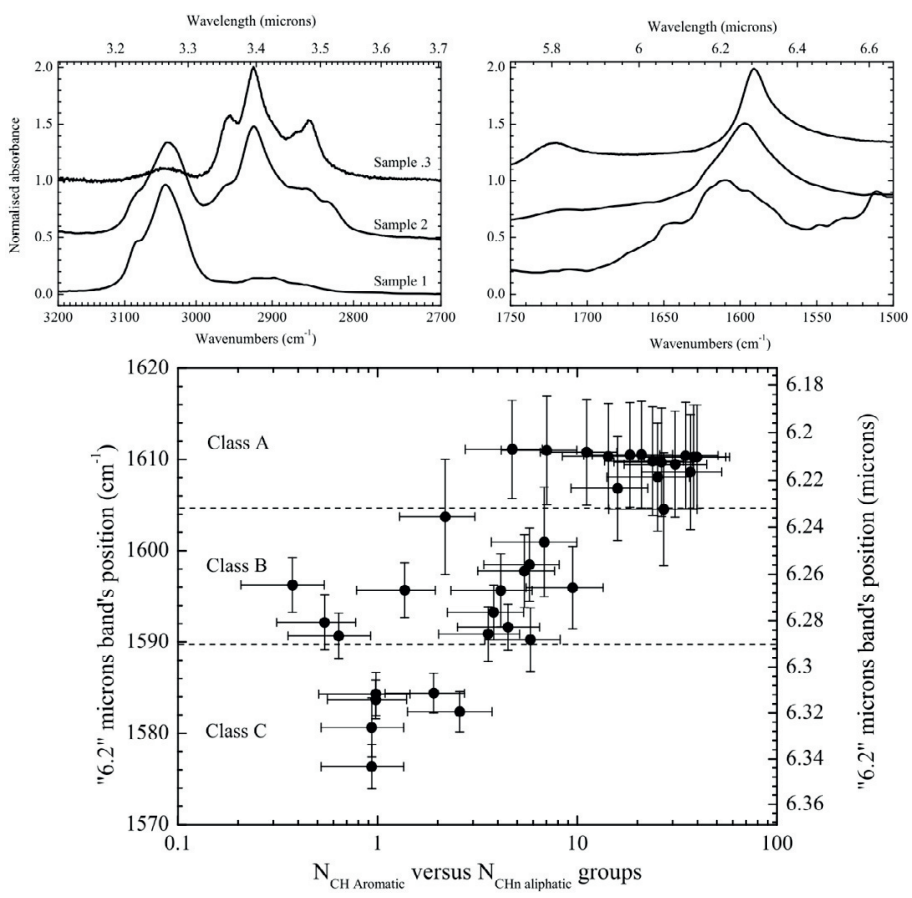

Fig. 4. Upper panel: infrared absorption spectra of laboratory soot samples with varying aliphatic to aromatic $\mathrm{C}-\mathrm{H}$ stretch content and associated $\mathrm{C}=\mathrm{C}$ mode region. Lower panel: evolution of the $\mathrm{C}=\mathrm{C}$ position in the various samples produced as a function of the aromatic to aliphatic ratio observed in the stretching mode region. Note that the total $\mathrm{H} / \mathrm{C}$ vary among the samples. The expected astrophysical classes range of $6.2 \mu \mathrm{m}$ positions are shown for comparison.

\subsection{A possible aromatic $\mathrm{C}=\mathrm{C}$ shift induced by aliphatics}

The sources with the "6.2 $\mu \mathrm{m}$ " feature lying at $6.3 \mu \mathrm{m}$ also display additional emission lines around 6.85 and $7.25 \mu \mathrm{m}$, as well as a more or less pronounced 
$3.4 \mu \mathrm{m}$ emission band on the side of the aromatic feature. Sloan et al. (2007) had proposed that the observed wavelength shifts are consistent with hydrocarbon mixtures containing both aromatic and aliphatic bonds. More recently, Acke et al. (2010) showed the same behaviour with a large set of Spitzer spectra (see also Acke, elsewhere in this volume). In order to understand carbon dust nucleation, and the observed astrophysical classes of materials, laboratory soot analogue spectra have been recorded for 50 distinct samples, using a premixed low pressure flame. Varying the flame combustion regime with respect to the stoechiometric conditions, and forcing various sooting conditions allow to produce many different carbonaceous soot materials (Pino et al. 2008). The resultant analogues are investigated by FTIR spectroscopy. This spectral analysis, applied to the band shape and position variations, was then used to interpret the diversity and evolution of the features in the astronomical spectra. Strong correlations were evidenced between the spectral regions characteristic of the $\mathrm{C}=\mathrm{C}$ and $\mathrm{C}-\mathrm{H}$ modes in these analogs. These shed light on the origin of the infrared emission features. In particular, the observed shift in the position of the $6.2-6.3 \mu \mathrm{m}$ band is shown to be a key tracer of the evolution of the aliphatic to aromatic component of carbonaceous dust. The produced materials have been characterised by other techniques (Raman spectroscopy, TEM, elemental analysis). These analyses suggest that there is a structural modification of the carbonaceous backbone by aliphatics. The evolution between these extreme positions $(6.2$ to $6.3 \mu \mathrm{m})$ thus appears as tracing the underlying carbon skeleton structure, linked to the presence of aliphatics in the network that favours the development of an alternative basic structural unit as compared to classical aromatic compounds.

\section{References}

Acke, B., \& van den Ancker, M.E., 2006, A\&A, 457, 171

Acke, B., Bouwman, J., Juhász, A., et al., 2010, ApJ, 718, 558

Allamandola, L.J., Sandford, S.A., \& Valero, G.J., 1988, Icarus, 76, 225

Allen, \& Wickramasenghe, 1981, Nature, 294, 239

Anders, E., \& Zinner, E., 1993, Meteoritics, 28, 490

Angus, J.C., \& Jansen, F., 1988, J. Vacuum Sci. Technol., 6, 1778

Bernstein, M.P., Sandford, S.A., Allamandola, L.J., Chang, S., \& Scharberg, M.A., 1995, ApJ, 454, 327

Butchart, I., McFadzean, A.D., Whittet, D.C.B., Geballe, T.R., \& Greenberg, J.M., 1986, A\&A, 154, L5

Chang, H.-C., Lin, J.-C., Wu, J.-Y., \& Chen, K.-H., 1995, J. Phys. Chem., 99, 11081

Chen, P.-S., Yang, X.-H., \& Shan, H.-G., 2010, Res. Astron. Astrophy., 10, 363

Chiar, J.E., \& Tielens, A.G.G.M., 2001, ApJ, 550, L207

Chiar, J.E., Adamson, A.J., Pendleton, Y.J., et al., 2002, ApJ, 570, 198

Dartois, E., Marco, O., Muñoz-Caro, G.M., et al., 2004, A\&A, 423, 549

Dartois, E., Muñoz Caro, G.M., Deboffle, D., Montagnac, G., \& D’Hendecourt, L., 2005, A\&A, 432, 895 
Dartois, E., et al., 2007, A\&A, 463, 635

Dartois, E., \& Muñoz-Caro, G.M., 2007, A\&A, 476, 1235

Duley, W.W., \& Williams, D.A., 1983, MNRAS, 205, 67P

Duley, W.W., Scott, A.D., Seahra, S., \& Dadswell, G., 1998, ApJ, 503, L183

Duley, W.W., 1994, ApJ, 430, L133

Ehrenfreund, P., Robert, F., D'Hendecourt, L., \& Behar, F., 1991, A\&A, 252, 712

Ferrari, A.C., \& Robertson, J., 2000, Phys. Rev. B, 61, 14095

Foing, B.H., \& Ehrenfreund, P., 1994, Nature, 369, 296

Fulara, J., Jakobi, M., \& Maier, J.P., 1993, Chemical Physics Letters, 211, 227

Furton, D.G., Laiho, J.W., \& Witt, A.N., 1999, ApJ, 526, 752

Gail, H.-P., \& Sedlmayr, E., 1999, A\&A, 347, 594

Geballe, T.R., 1997, From Stardust to Planetesimals, 122, 119

Geballe, T.R., Chiar, J., Pendleton, Y.J., \& Tielens, A.G.G.M., 1998, Astrophys. Space Sci., 255, 457

Geballe, T.R., Mason, R.E., Rodríguez-Ardila, A., \& Axon, D.J., 2009, ApJ, 701, 1710

Godard, M., \& Dartois, E., 2010, A\&A, forthcoming

Goto, M., Maihara, T., Terada, H., Kaito, C., Kimura, S., \& Wada, S., 2000, Astron. Astrophys. Suppl., 141, 149

Goto, M., et al., 2003, ApJ, 589, 419

Goto, M., et al., 2007, ApJ, 662, 389

Goto, M., et al., 2009, ApJ, 693, 610

Greenberg, J.M., Li, A., Mendoza-Gomez, C.X., et al., 1995, ApJ, 455, L177

Guillois, O., Ledoux, G., \& Reynaud, C., 1999, ApJ, 521, L133

Habart, E., Testi, L., Natta, A., \& Carbillet, M., 2004, ApJ, 614, L129

Herbig, G.H., 2000, ApJ, 542, 334

Hill, H.G.M., Jones, A.P., \& D'Hendecourt, L.B., 1998, A\&A, 336, L41

Hony, S., 2002, Ph.D. Thesis

Hudgins, D.M., Bauschlicher, C.W., Jr., \& Allamandola, L.J., 2005, ApJ, 632, 316

Imanishi, M., 2006, AJ, 131, 2406

Imanishi, M., Nakagawa, T., Ohyama, Y., et al., 2008, PASJ, 60, 489

Joblin, C., Tielens, A.G.G.M., Allamandola, L.J., \& Geballe, T.R., 1996, ApJ, 458, 610

Jones, T.J., Hyland, A.R., \& Allen, D.A., 1983, MNRAS, 205, 187

Jones, A.P., 1990, MNRAS, 247, 305

Koike, C., Wickramasinghe, N.C., Kano, N., et al., 1995, MNRAS, 277, 986

Lee, W., \& Wdowiak, T.J., 1993, ApJ, 417, L49

Mason, R.E., Wright, G., Pendleton, Y., \& Adamson, A., 2004, ApJ, 613, 770

Mason, R.E., Geballe, T.R., Packham, C., et al., 2006, ApJ, 640, 612

McFadzean, A.D., Whittet, D.C.B., Bode, M.F., Adamson, A.J., \& Longmore, A.J., 1989, MNRAS, 241, 873

Mennella, V., Brucato, J.R., Colangeli, L., \& Palumbo, P., 1999, ApJ, 524, L71

Mennella, V., Brucato, J.R., Colangeli, L., \& Palumbo, P., 2002, ApJ, 569, 531

Moutou, C., Sellgren, K., Verstraete, L., \& Léger, A., 1999, A\&A, 347, 949 
Ott, U., 1993, Nature, 364, 25

Patzer, A.B.C., Chang, C., John, M., Sedlmayr, E., \& Sülzle, D., 2003, Astron. Nachr. Suppl., 324, 20

Pendleton, Y.J., \& Allamandola, L.J., 2002, ApJS, 138, 75

Pendleton, Y.J., Sandford, S.A., Allamandola, L.J., Tielens, A.G.G.M., \& Sellgren, K., 1994, ApJ, 437, 683

Peeters, E., Hony, S., Van Kerckhoven, C., et al., 2002, A\&A, 390, 1089

Pino, T., et al., 2008, A\&A, 490, 665

Pirali, O., Vervloet, M., Dahl, J.E., et al., 2007, ApJ, 661, 919

Risaliti, G., et al., 2006, MNRAS, 365, 303

Sakata, A., Wada, S., Onaka, T., \& Tokunaga, A.T., 1990, ApJ, 353, 543

Sandford, S.A., Allamandola, L.J., Tielens, A.G.G.M., et al., 1991, ApJ, 371, 607

Sandford, S.A., Pendleton, Y.J., \& Allamandola, L.J., 1995, ApJ, 440, 697

Schnaiter, M., Mutschke, H., Dorschner, J., Henning, T., \& Salama, F., 1998, ApJ, 498, 486

Scott, A.D., Duley, W.W., \& Jahani, H.R., 1997, ApJ, 490, L175

Sellgren, K., Werner, M.W., \& Ingalls, J.G., 2009, Amer. Astron. Soc. Meet. Abs., 214, \#402.12

Sellgren, K., Werner, M.W., Ingalls, J.G., et al., 2010, ApJ, 722, L54

Sloan, G.C., et al., 2007, ApJ, 664, 1144

Szczerba, R., Siódmiak, N., \& Szyszka, C., 2005, Planet. Nebulae Astron. Tools, 804, 214

Tielens, A.G.G.M., Wooden, D.H., Allamandola, L.J., Bregman, J., \& Witteborn, F.C., 1996, ApJ, 461, 210

van Diedenhoven, B., Peeters, E., Van Kerckhoven, C., et al., 2004, ApJ, 611, 928

Volk, K., Kwok, S., Hrivnak, B., \& Szczerba, R., 2001, Astrophys. Space Sci. Libr., 265, 323 
